\title{
The Spitzer spectroscopic survey of the Small Magellanic Cloud: polycyclic aromatic hydrocarbon emission from SMC star-forming regions
}

\author{
Karin M. Sandstrom ${ }^{1}$, Alberto D. Bolatto ${ }^{2}$, Snežana Stanimirović ${ }^{3}$, \\ J. D. T. Smith ${ }^{4}$, Jacco Th. van Loon ${ }^{5}$ and Adam K. Leroy ${ }^{6}$ \\ ${ }^{1}$ Dept. of Astronomy, University of California, Berkeley, 601 Campbell Hall, \\ Berkeley CA 94720 USA \\ email: karin@astro.berkeley.edu \\ ${ }^{2}$ Dept. of Astronomy \& Laboratory for Millimeter-wave Astronomy, University of Maryland, \\ College Park, College Park, MD 20742 USA \\ ${ }^{3}$ Dept. of Astronomy, University of Wisconsin, Madison, Madison, WI 53706 USA \\ ${ }^{4}$ Ritter Astrophysical Research Center, University of Toledo Toledo, OH 43603 USA \\ ${ }^{5}$ Astrophysics Group, Lennard-Jones Laboratories, Keele University, \\ Staffordshire ST5 5BG, UK \\ ${ }^{6}$ Max-Planck-Institut für Astronomie, Königstuhl 17, D-69117 Heidelberg, Germany
}

\begin{abstract}
Because of its proximity, the Small Magellanic Cloud provides a unique opportunity to map the polycyclic aromatic hydrocarbon $(\mathrm{PAH})$ emission from photo-dissociation regions (PDRs) in a low-metallicity $(12+\log (\mathrm{O} / \mathrm{H}) \sim 8)$ galaxy at high spatial resolution in order to learn about their abundance and physical state. We present mid-IR spectral mapping observations of star-forming regions in the Small Magellanic Cloud obtained as part of the Spitzer Spectroscopic Survey of the SMC ( $\left.\mathrm{S}^{4} \mathrm{MC}\right)$ project. These observations allow us to map the distribution of PAH emission in these regions and the measure the variation of $\mathrm{PAH}$ band strengths with local physical conditions. In these proceedings we discuss preliminary results on the physical state of the PAHs, in particular their ionization fraction.
\end{abstract}

Keywords. dust, extinction, HII regions, ISM: molecules, galaxies: individual (SMC), Magellanic Clouds, infrared: ISM

\section{Introduction}

Dust grains span a range of sizes from the $\sim 0.1 \mu \mathrm{m}$ "classical" grains which provide most of the optical extinction to the few $\AA$-sized polycyclic aromatic hydrocarbons (PAHs), which straddle the line between very small dust grains and large molecules. PAHs are ubiquitous in the Milky Way, as shown by the widely-observed mid-IR emission bands (for a recent review see Tielens 2008). PAHs have significant roles in ISM heating, particularly in photodissociation regions (PDRs) where PAHs can be the dominant source of photoelectrons; grain surface chemistry; and charge balance, particularly in low-UV flux regions where they can efficiently mediate charge exchange reactions (Bakes \& Tielens 1998). The physical state of PAHs is intimately tied to their roles in the ISM. For example, the charge state of PAHs in a PDR determines the efficiency of the photoelectric effect in the far-UV dominated regions of the PDR. The physical state of PAHs also has a direct impact on their destruction by UV radiation fields. PAHs that are small, highly 
ionized, and/or dehydrogenated are more susceptible to photo-dissociation (Allain et al. 1996).

Observations with Spitzer and ISO have convincingly demonstrated that there is a deficiency in PAH emission from low-metallicity galaxies (Madden et al. 2006; Engelbracht et al. 2005, 2008; Draine et al. 2007). This deficit has been attributed to a variety of causes: delayed injection of PAHs into the ISM because of the long lifetimes of their evolved star progenitors (Galliano et al. 2008a), destruction of PAHs by supernova shock waves (O'Halloran et al. 2006) and/or destruction by harder and more intense UV radiation fields (Madden et al. 2006). In the SMC, the deficiency of PAH emission has been observed in the 8 -to- $24 \mu \mathrm{m}$ ratio in the Southwest Bar region by Bolatto et al. (2007). The SMC is a particularly interesting object for studying PAH emission from low-metallicity galaxies because its metallicity of $12+\log (\mathrm{O} / \mathrm{H}) \sim 8$ puts it right at the observed transition where more metal-rich galaxies show normal PAH emission and more metal-poor galaxies have mid-IR colors and spectra consistent with litte to no PAH emission.

If PAHs are being destroyed by more intense UV radiation fields in low-metallicity galaxies, we may expect to see some signature of this process in their ionization state or size distribution. These properties can be diagnosed by examining the ratios of the mid-IR emission bands (Allamandola et al. 1999). Ionized PAHs exhibit higher ratios of $\mathrm{C}-\mathrm{C} / \mathrm{C}-\mathrm{H}$ band strengths compared to neutral PAHs. For instance, the ratio of the 6.2 $\mu \mathrm{m}$ and $7.7 \mu \mathrm{m} \mathrm{C}-\mathrm{C}$ bands versus the $11.3 \mu \mathrm{m} \mathrm{C}-\mathrm{H}$ band traces the ionization of the PAHs, (see recent work by Galliano et al. 2008b). In the following, we present observations of the mid-IR emission bands from PAHs in star-formation regions in the SMC obtained as part of the Spitzer Spectroscopic Survey of the SMC ( $\left.\mathrm{S}^{4} \mathrm{MC}\right)$. We discuss preliminary results on the ionization state of PAHs and the variations of the mid-IR band ratios.

\section{Observations \& data reduction}

$\mathrm{S}^{4} \mathrm{MC}$ covered six of the major star-forming regions of the SMC with spectral mapping observations using the low-resolution orders (SL and LL) of the InfraRed Spectrograph (IRS) on Spitzer. The maps were fully sampled spatially and had integration times per slit of 14 and 30 seconds for SL and LL orders, respectively. The maps cover typically $\sim 70 \operatorname{arcmin}^{2}$ in LL and $\sim 14 \operatorname{arcmin}^{2}$ in SL. The SL map of the SMC B1 region covers $\sim 6 \operatorname{arcmin}^{2}$ with 60 second integrations per slit in order to get higher signal-to-noise in this unique region. A dedicated "off" position was used to remove the Galactic and zodiacal light foregrounds from the observations. A more detailed discussion of the $\mathrm{S}^{4} \mathrm{MC}$ observations and data reduction can be found in Sandstrom et al. (2008).

The spectral maps were calibrated and assembled in IDL using the Cubism package (Smith et al. 2007). In the following we discuss only the data in the SL orders, which range from 5-14 $\mu \mathrm{m}$. Each wavelength slice of the cubes was convolved to match the lowest resolution point spread function of the SL observations (i.e. that of the $\lambda=14.74 \mu \mathrm{m}$ ) using convolution kernels derived from the predictions of sTinyTim $\dagger$. More information on the convolution kernels can be found in Sandstrom et al. (2008). The cubes were aligned to match the SL1 pixel grid. The PAH feature strengths were measured in IDL using the program Pahfit (Smith et al. 2007). Figure 1 shows the spectrum of the peak of $\mathrm{PAH}$ emission in SMC B1 with the best-fit results of Pahfit overplotted.

$\dagger$ http://ssc.spitzer.caltech.edu/archanaly/contributed/stinytim/index.html 


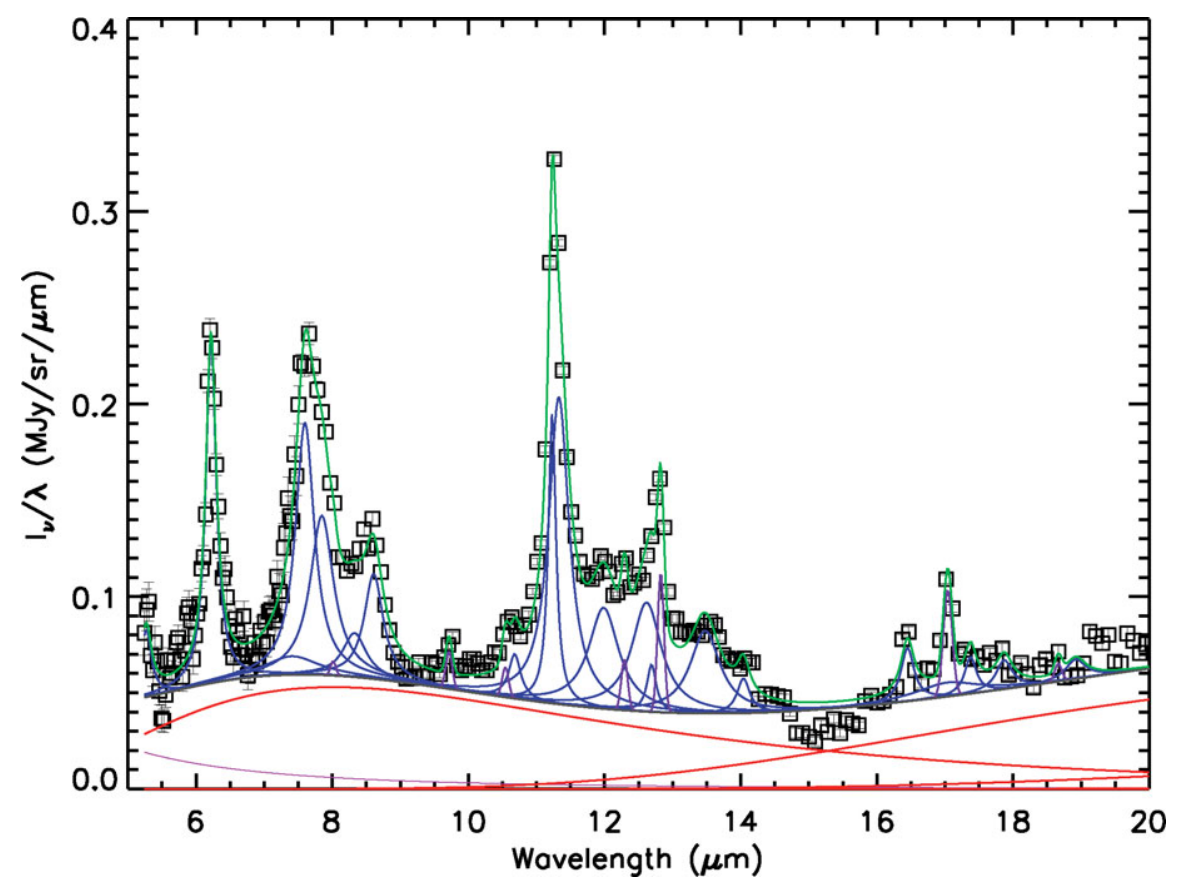

Figure 1. The PAH emission spectrum of the brightest position in SMC B1 overlayed with the results of Pahfit. Pahfit decomposes the spectrum into contributions from starlight continuum, dust continuum, emission lines and PAH band features.

\section{Results}

\subsection{SMC B1 molecular cloud}

SMC B1\#1 is a quiescent molecular cloud in the Southwest Bar of the SMC. There are no $\mathrm{H}$ II regions or massive stars in its vicinity, so the dust is heated by a combination of the overall SMC radiation field and possibly a few nearby stars. The first detection of PAH emission from the SMC was from this region by Reach et al. (2000) using ISO observations. They noted the unusual strength of the $11.3 \mu \mathrm{m}$ feature relative to the $7.7 \mu \mathrm{m}$ feature. We also observe a low ratio of the $7.7 / 11.3$ bands in this region. Figure 2 shows a map of the integrated intensity of the $11.3 \mu \mathrm{m}$ band with a few selected regions highlighted. Figure 3 shows the average spectrum in the highlighted regions.

The 7.7/11.3 band ratio in SMC B1 has an average value of $\sim 2$ and does not show any significant spatial variations. Comparing these ratios to those in Galliano et al. (2008b) we find that the PAH emission from SMCB1 is consistent with a low ionization level.

\subsection{N 66 star-forming region}

$\mathrm{N} 66$ is the most luminous $\mathrm{H}$ II region in the SMC containing $33 \mathrm{O}$ stars (Massey et al. 1989). PAH emission from N 66 has been studied previously by Contursi et al. (2000). The higher angular resolution of Spitzer allows us to observe PAH emission tracing out the filamentary structures of the H II region. Figure 4 shows the integrated intensity of the $11.3 \mu \mathrm{m}$ feature in this region with boxes showing the regions over which we have extracted spectra. Figure 5 shows a few of those spectra.

The 7.7/11.3 band ratio shows a wider range of variation in N 66 than in SMC B1. This is consistent with some regions having a higher PAH ionization fraction than achieved in SMC B1. However, we also observe band ratios as low as those observed in SMC B1. This 


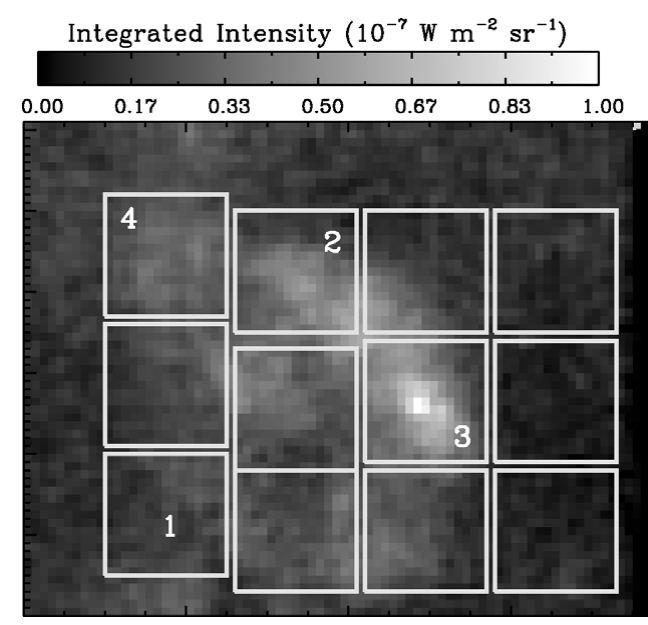

Figure 2. This figure shows the integrated intensity of the $11.3 \mu \mathrm{m} \mathrm{PAH}$ feature in the SMC B1 region. In order to increase the signal-to-noise we have extracted spectra from the cube in $30^{\prime \prime}$ boxes. The spectra from the numbered boxes are plotted in Figure 3 .

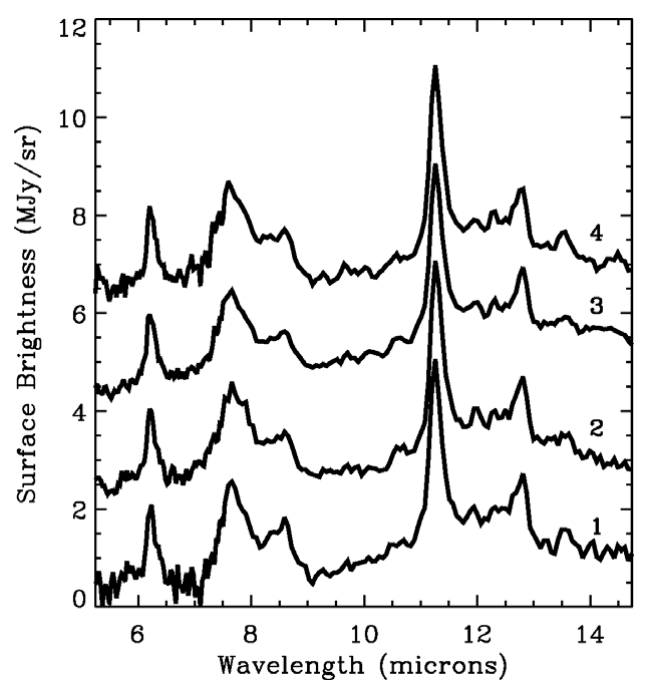

Figure 3. Spectra from the numbered boxes in Figure 2. The spectra are normalized to the $11.3 \mu \mathrm{m}$ band and then shifted by $2 \mathrm{MJy}$ $\mathrm{sr}^{-1}$ for clarity. The band strengths in SMC B1 show little variation across the region.

is particularly interesting because previous theoretical studies have had difficulty reproducing the band ratios in SMCB1 without adjusting the intrinsic band strengths (Li \& Draine 2002). Excess hydrogenation and/or excitation by reddened starlight (rather than the average SMC radiation field) have been proposed as explanations for the band ratios in SMC B1. Seeing similar 7.7/11.3 ratios in both N 66 and SMC B1, two very different regions, suggests that different intrinsic band strengths may be the best explanation.

\subsection{Band ratio variations}

In addition to variations in the $7.7 / 11.3$ band ratios, we observe variations in the ratios of different $\mathrm{C}-\mathrm{C}$ bands as well, something that has not been observed in previous studies of more metal-rich galaxies (Galliano et al. 2008b). Figure 6 shows the variations in the $7.7 / 11.3$ and 7.7/6.2 ratio, the latter of which is a ratio between two $\mathrm{C}-\mathrm{C}$ emission bands. In our observations these band ratios appear to be positively correlated in N 66 and N 83, with $\mathrm{N} 66$ offset towards larger 7.7/6.2 ratios. It is interesting that the band ratios from these regions seem to fall in distinct groups on the plot, suggesting different physical states for the PAHs, possibly related to different processing by the UV radiation field in each region.

\section{Summary \& conclusions}

We detect emission from PAHs in all of the regions mapped by the $\mathrm{S}^{4} \mathrm{MC}$ project. Although it has been shown that $\mathrm{PAH}$ emission is deficient in the SMC relative to more metal-rich galaxies Bolatto et al. (2007), PAHs are not absent from the SMC. In this proceedings we present a preliminary investigation of the band ratio variations. We find 7.7/11.3 ratios that are consistent with a range of ionization states for the PAHs across the SMC. In the quiescent molecular cloud SMC B1 the 7.7/11.3 ratio indicates a lower ionization level. Our observations of the band ratios in SMC B1 agree with the values determined by Reach et al. (2000). In addition, however, we see similarly low $7.7 / 11.3$ 


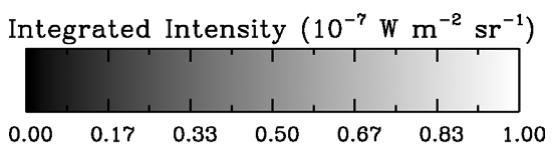

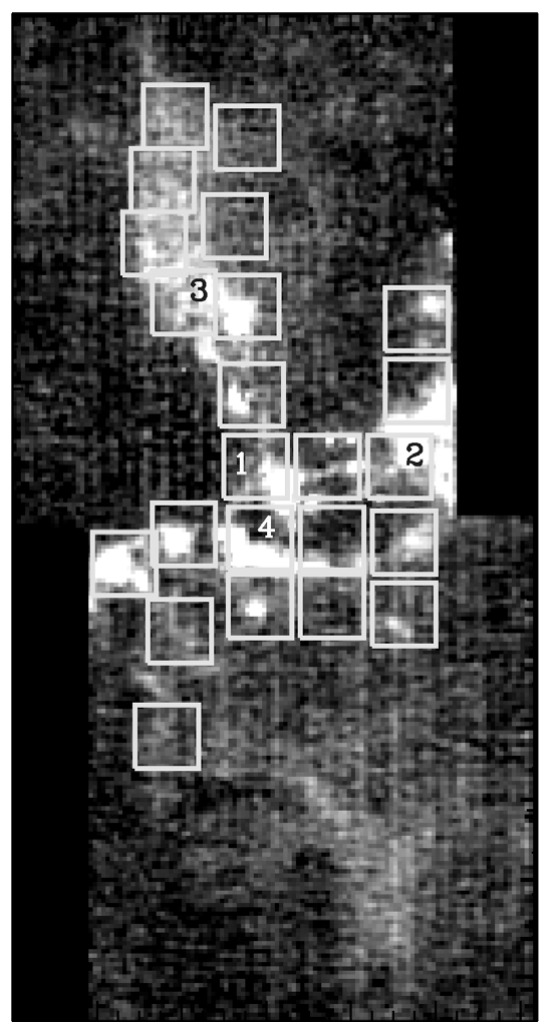

Figure 4. Integrated intensity of the $11.3 \mu \mathrm{m}$ band in $\mathrm{N} 66$.

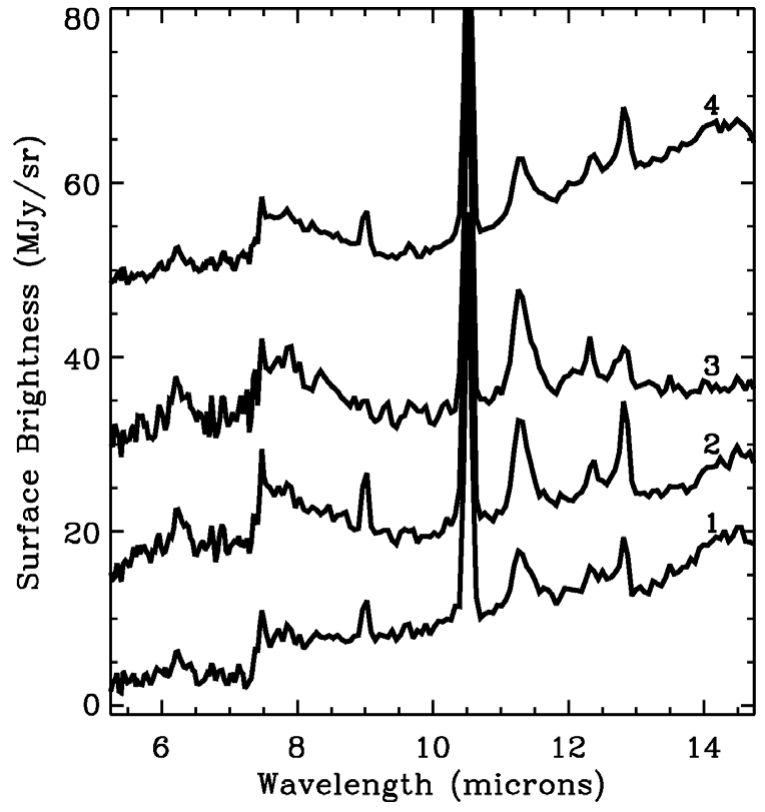

Figure 5. Spectra of selected regions from N 66, normalized to the $11.3 \mu \mathrm{m}$ band strength and then shifted by $15 \mathrm{MJy} \mathrm{sr}^{-1}$. The strong emission line at $10.5 \mu \mathrm{m}$ is from $[\mathrm{S} I V]$.

ratios even in $\mathrm{N} 66$, suggesting that the intrinsic band strengths may be different in the SMC and the Milky Way, rather than SMC B1 having excess PAH hydrogenation or some other unique excitiation conditions. We also observe variations in the 7.7/6.2 ratio which are positively correlated with the $7.7 / 11.3$ ratio. These observations will be explored further in an upcoming paper (Sandstrom et al. 2009, in preparation).

\section{References}

Allain, T., Leach, S., \& Sedlmayr, E. 1996, A\& A, 305, 616

Allamandola, L. J. and Hudgins, D. M., \& Sandford, S. A. 1999, ApJ, 511, L115

Bakes, E. L.O. \& Tielens, A. G. G. M. 1998, ApJ, 499, 258

Bolatto, A. D., Simon, J. D., Stanimirović, S., et al. 2007, ApJ, 655, 212

Contursi, A., Lequeux, J., Cesarsky, D., et al. 2000, A\&SA, 362, 310

Draine, B. T., Dale, D. A., Bendo, G., et al. 2007, ApJ, 663, 866

Engelbracht, C. W., Gordon, K. D., Rieke, G. H., Werner, M. W., Dale, D. A., \& Latter, W. B. 2005, ApJ, 628, L29

Engelbracht, C. W., Rieke, G. H., Gordon, K. D., Smith, J.-D. T., Werner, M. W., Moustakas, J., Willmer, C. N. A., \& Vanzi, L. 2008, ApJ, 678, 804

Galliano, F., Dwek, E., \& Chanial, P. 2008a, ApJ, 672, 214 


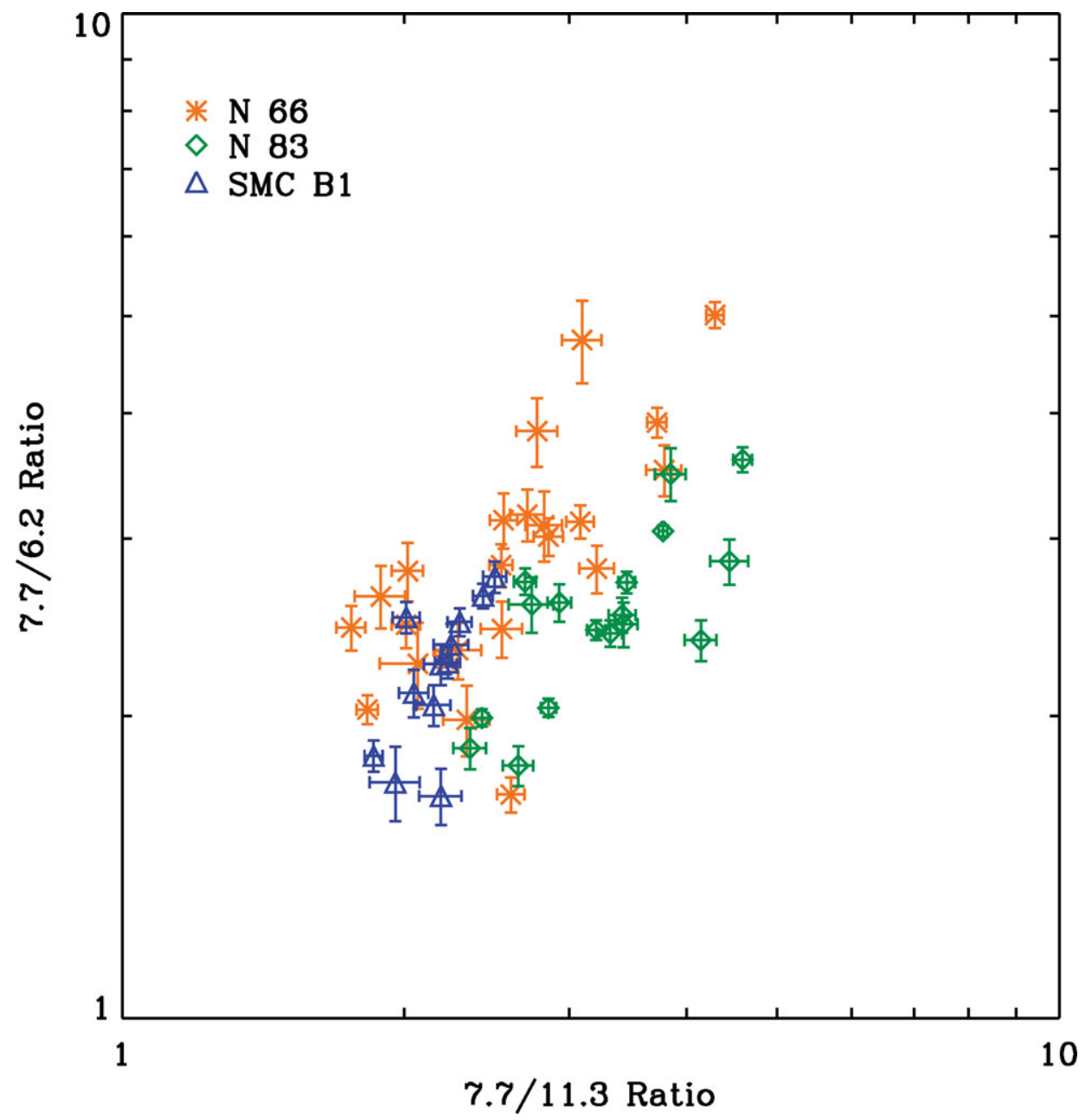

Figure 6. A plot of the $7.7 / 6.2$ vs. 7.7/11.3 ratio in the $\mathrm{N} 66, \mathrm{~N} 83$ and SMC B1 regions (the points are extracted from the boxes shown on previous plots). The ratios are positively correlated in $\mathrm{N} 66$ and $\mathrm{N} 83$.

Galliano, F., Madden, S. C., Tielens, A. G. G. M., Peeters, E., \& Jones, A. P. 2008b, ApJ, 679, 310

Li, A. \& Draine, B. T. 2002, ApJ, 576, 762

Madden, S. C., Galliano, F., Jones, A. P., \& Sauvage, M. 2006, A\& A, 446, 877

Massey, P., Parker, J. W., \& Garmany, C. D. 1989, AJ, 98, 1305

O'Halloran, B., Satyapal, S., \& Dudik, R. P. 2006, ApJ, 641, 795

Reach, W. T., Boulanger, F., Contursi, A., \& Lequeux, J. 2000, A\&A $A$, 361, 895

Sandstrom, K. M., Bolatto, A. D., Stanimirović, S., van Loon, J.Th, \& Smith, J. D. T. 2008, ApJ, submitted [ArXiv:0810.2803]

Smith, J. D. T., Armus, L., \& Dale, D. A. 2007, PASP, 119, 1133

Tielens, A. G. G. M. 2008, ARAA, 46, 289 